\title{
Reisefähigkeit ist Teil der Lebensqualität
}

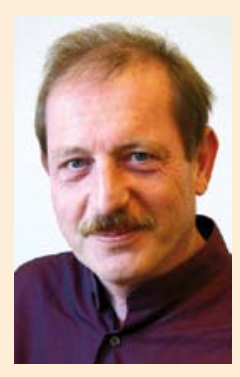

Mimman

Liebe Kolleginnen und Kollegen,

es ist unstrittig, dass der Fortschritt der Medizin in Verbindung mit verbesserten Lebensbedingungen zu einer Verlängerung der Lebenszeit von Menschen führt. Die reine Verlängerung der Lebenszeit, das Überleben als solches, steht jedoch in hochentwickelten Industriegesellschaften nicht mehr im Zentrum medizinischen Handelns. Vielmehr kommt es darauf an, im Ergebnis ein möglichst hohes Maß an Lebensqualität bis in das hohe Alter hinein zu gewährleisten. Gleiches gilt für Menschen mit chronischen Erkrankungen, völlig unabhängig des Lebensalters.

Die Möglichkeit zu Reisen stellt dabei ein so wesentliches Element der Lebensqualität dar, dass sie auch für Menschen mit gesundheitlichen Einschränkungen ohne erhöhtes Risiko gegeben sein muss. Dies setzt zunächst voraus, dass sich Reiseveranstalter und Transportunternehmen auf gesundheitliche Einschränkungen ihrer Kunden einstellen und eine gewisse „Grundsicherung“ gewährleisten. Hier muss die Medizin Wege aufzeigen. Eine zentrale Bedeutung kommt allerdings dem behandelnden Arzt des chronisch Erkrankten zu, der beratungsfähig sein muss, um die gesundheitliche Einschränkung des jeweiligen Patienten mit seiner Reiseabsicht so in Einklang zu bringen, dass diese weder unnötig verhindert wird, noch mit einem unkalkulierbaren Risiko verbunden ist.

Das vorliegende Schwerpunktheft der FTR widmet sich genau diesem Themenkreis. Jörg Siedenburg gibt mit seinem Beitrag „Flugreisen mit gesundheitlichen Einschränkungen“ einen generellen Überblick beratungsrelevanter medizinischer Aspekte im Luftverkehr. Jürgen Ringwald, Piotr Kuta und Reinhold Eckstein vermitteln in ihrem Artikel „Hämophilie und Reisen“ detailliert diejenigen Informationen, die für die Beratung von Hämophiliepatienten bedeutungsvoll sind. Der großen Gruppe der Diabeteskranken widmet sich Burkard Rieke in seinem Beitrag „Reisen mit Diabetes“, für die eine eingeschränkte Mobilität gewöhnlich kein Reisehindernis darstellt, jedoch die veränderten Lebensbedingungen auf Reisen mit besonderen Risiken verbunden sind. Einem nicht unbedeutenden technischen Aspekt widmet sich Christoph Sevenich mit seinen Informationen über „Implantierte Herzschrittmacher und Defibrillatoren auf Reisen“, da nicht nur eine Dauermedikation, sondern auch implantierte Medizingeräte bei Reisenden berücksichtigt werden müssen.

Ich hoffe, dass auch diese Ausgabe der FTR wieder nützliche Informationen für Sie bereithält und wünsche ein frohes Weihnachtsfest und ein glückliches neues Jahr.

Mit freundlichen Grüßen aus Kiel

Ihr Dr. Stefan Neidhardt 